\title{
Interleukin- $1 \beta$ Induces a Reactive Astroglial Phenotype via Deactivation of the Rho GTPase-Rock Axis
}

\author{
Gareth R. John, ${ }^{1,2}$ Lanfen Chen, ${ }^{2}$ Mark A. Rivieccio, ${ }^{2}$ Carmen V. Melendez-Vasquez, ${ }^{4}$ Adam Hartley, ${ }^{3}$ and \\ Celia F. Brosnan ${ }^{2,3}$ \\ ${ }^{1}$ Corinne Goldsmith Dickinson Center for Multiple Sclerosis, Department of Neurology, Mount Sinai School of Medicine, New York, New York 10029, \\ Departments of ${ }^{2}$ Pathology and ${ }^{3}$ Neuroscience, Albert Einstein College of Medicine, Bronx, New York 10461, and ${ }^{4}$ Skirball Institute of Biomolecular \\ Medicine, New York University School of Medicine, New York, New York 10016
}

The cytokine interleukin-1 $\beta$ (IL-1 $\beta$ ) is critical to the formation of an astrocytic scar after CNS injury, but the mechanisms by which it induces a reactive phenotype remain unresolved. Here, we show that IL- $1 \beta$ regulates the phenotype of astrocytes via deactivation of the Rho GTPase-Rho kinase (ROCK) pathway, which governs cellular morphology and migration via effects on F-actin and its interactions with focal adhesions, nonmuscle myosin, and microvillar adapter proteins of the ezrin-radixin-moesin (ERM) family. We found that IL- $1 \beta$ induced cortical reorganization of F-actin and dephosphorylation of focal adhesion kinase, myosin light chain 2, and myosin phosphatase targeting subunit 1 in primary human astrocytes, and that all of these effects were mimicked by Rho-ROCK pathway blockade. We also found that IL- $1 \beta$ conversely potentiated ERM phosphorylation, and that this effect was mediated via a Rho-ROCKindependent mechanism. Next, we used a rhotekin pulldown assay to confirm directly that IL-1 $\beta$ deactivates Rho, and further demonstrated that a constitutively active Rho construct rescued astrocytes from developing an IL-1 $\beta$-induced reactive phenotype. These data implicate cytokine regulation of the Rho-ROCK pathway in the generation of a reactive astrogliosis, and we suggest that interventions targeted at this level may facilitate manipulation of the glial scar in inflammatory disorders of the human CNS.

Key words: astrocyte; inflammation; interleukin; myosin; focal adhesions; RhoA

\section{Introduction}

Astrocytes comprise the largest glial population in the mammalian brain, but our understanding of their fundamental properties and functions remains incomplete. Evidence would suggest that they are involved in provision of metabolic substrates for neurons (Voutsinos-Porche et al., 2003), maintenance of the extracellular ionic environment and $\mathrm{pH}$ (Fields and StevensGraham, 2002), and uptake of neurotransmitters (Danbolt, 2001). They interact with one another to form large networks (John et al., 1999), and associate with neurons, oligodendrocytes and endothelia: the latter interaction is believed to lead to the creation and maintenance of the blood-brain barrier (Janzer and Raff, 1987).

Injury to the CNS results in a characteristic astroglial response, referred to as an astrocytic scar. Astrocytes within the scar undergo a profound phenotypic change, and evidence from transgenic and gene-targeting studies suggests that these reactive cells are central regulators of the inflammatory response in the

\footnotetext{
Received 0ct. 23, 2003; revised Jan. 21, 2004; accepted Jan. 23, 2004.

This work was supported by the Jayne and Harvey Beker Research Program in Multiple Sclerosis (G.R.J.), by National Multiple Sclerosis Society Grant RG3444 (G.R.J.), and by United States Public Health Service Grants NS046620 (G.R.J.), NS40137 (C.F.B.), and NS11920 (C.F.B.). We thank Dr. Karen Weidenheim and Dr. Bradley Poulos (Human Fetal Tissue Repository), Dr. Sunhee Lee and Wa Shen (Department of Pathology), and Michael Cammer, Frank Macaluso, and Leslie Gunther (Analytical Imaging Facility) at the Albert Einstein College of Medicine.

Correspondence should be addressed to Dr. Gareth John, Annenberg Building 14-86, Box 11-37, Mount Sinai School of Medicine, 1 Gustave L. Levy Place, New York, NY 10029. E-mail: gareth.john@mssm.edu.

DOI:10.1523/JNEUROSCI.4789-03.2004

Copyright $\odot 2004$ Society for Neuroscience $\quad 0270-6474 / 04 / 242837-09 \$ 15.00 / 0$
}

CNS. For example, specific ablation of scar-forming reactive astrocytes in a transgenic GFAP-thymidine kinase model is associated with dramatic alterations in the response of the CNS to injury, including a prolonged 25 -fold increase in leukocyte infiltration, failure of blood-brain barrier repair, substantial neuronal degeneration that appears to be glutamate-dependent, and a pronounced increase in local neurite outgrowth (Bush et al., 1999).

Although these results suggest a functional role for the astrocytic scar in regulating CNS repair, the mechanisms that generate a reactive astroglial phenotype are still incompletely understood. However, studies performed both in vivo and in vitro have identified at least some of the factors that contribute to scar formation. These studies have shown that, of the inflammatory mediators known to be present after CNS injury, only one, the cytokine interleukin-1 $\beta$ (IL-1 $\beta$ ), appears critical for the induction of a reactive astroglial phenotype (Giulian et al., 1988; Herx and Yong, 2001). This cytokine is a potent regulator of gene transcription, exerting its effects via activation of transcription factors including NF- $\kappa \mathrm{B}$ and AP-1 (Ghosh and Karin, 2002; O'Neill, 2002), and we recently studied its effects on astrocytic gene expression patterns using transcriptome-scale screening (John et al., 2002). However, although our study recorded the effects of IL- $1 \beta$ on multiple gene families, it did not reveal the structural phenotype of a reactive astrocyte. We have therefore explored the potential role of post-translational effects in this response, and we have identified the molecular events involved and their underly- 
ing mechanism. We have found that IL- $1 \beta$ regulates the phenotype of astrocytes via an interaction with a pathway mediated via small GTPases of the Rho subfamily and their downstream effector Rho kinase (ROCK). This pathway regulates the morphology and migration of mammalian cells via effects on F-actin and its interactions with focal adhesions, non-muscle myosins, and ezrin-radixin-moesin (ERM) adapter proteins (Amano et al., 2000 ). Our data show that IL- $1 \beta$ regulates all of these events in human astrocytes, and further that its effects on actomyosin and focal adhesions are mimicked by blockade of ROCK. We also demonstrate directly that IL- $1 \beta$ deactivates Rho, and that a constitutively active Rho construct rescues astrocytes from developing an IL- $1 \beta$-induced reactive phenotype.

\section{Materials and Methods}

Glial cell cultures. Tissue collection was approved by the Institutional Clinical Review Committee. Human fetal brain astrocyte cultures were established as previously described (Liu et al., 1996).

Cytokines and growth factors. Recombinant human IL- $1 \beta$ (Peprotech, Rocky Hill, NJ) was used at $10 \mathrm{ng} / \mathrm{ml}$, based on dose-response studies that we and others have performed previously on IL-1-mediated signal transduction in human fetal astrocytes (Liu et al., 2000) and multiple other cell types (Delhase et al., 1999).

Inhibitors. The selective ROCK inhibitors Y-27632 and HA1077 (Uehata et al., 1997) were obtained from Calbiochem (San Diego, CA). They were used at final concentrations of 10 and $20 \mu \mathrm{M}$ respectively, as has been previously published (Suzuki et al., 1999; Verhoef et al., 2003).

Antibodies. Rabbit anti-phospho-myosin light chain 2 (Ser19, or Thr18, Ser19), anti-phospho-ezrin (Thr567)/radixin (Thr564)/moesin (Thr558), anti-total ezrin/radixin/moesin, anti-phospho-Rac1/cdc42 (Ser71), and anti-phospho-focal adhesion kinase (FAK) (Tyr576/577) were obtained from Cell Signaling (Beverly, MA). Rabbit anti-total FAK, and mouse anti-vascular cellular adhesion molecule-1 (VCAM-1) were obtained from Santa Cruz Biotechnology (Santa Cruz, CA). Mouse antiintercellular adhesion molecule-1 (ICAM-1) was obtained from Santa Cruz and from R \& D Systems (Minneapolis, MN). Mouse anti-vinculin and anti-actin antibodies were obtained from Chemicon (Temecula, CA), and rabbit anti-Rho (-A, -B, -C) GTPase and anti-phospho-myosin phosphatase targeting subunit 1 (Thr696) were obtained from Upstate Biotechnology (Lake Placid, NY). Mouse anti-bromodeoxyuridine (BrdU) was obtained from BD Biosciences (San Diego, CA).

Scanning electron microscopy. Astrocyte cultures grown on $10 \mathrm{~mm}$ diameter glass coverslips were fixed $15 \mathrm{~min}$ in $2.5 \%$ glutaraldehyde, $0.1 \mathrm{~m}$ sodium cacodylate, $0.2 \mathrm{M}$ sucrose, $5 \mathrm{~mm} \mathrm{MgCl}_{2}, \mathrm{pH} 7.4$, dehydrated through a graded series of ethanols, then subjected to critical point drying using liquid carbon dioxide in a Tousimis Samdri 795 critical point drier (Rockville, MD). Samples were sputter-coated with gold-palladium in a Denton Vacuum Desk-2 sputter-coater (Cherry Hill, NJ), and were examined using a JEOL JSM6400 scanning electron microscope (Peabody, MA), using an accelerating voltage of $10 \mathrm{kV}$.

Confocal microscopy. Astrocyte cultures grown on glass confocal plates (Mat-Tek, Ashland, MA) and treated as described were fixed $15 \mathrm{~min}$ in PBS 4\% paraformaldehyde, rinsed two times in PBS and once in PBS $0.1 \%$ glycine, then blocked in PBS $0.3 \%$ Triton X-100 10\% goat serum 30 min and incubated with primary antibodies $(1: 100)$ in blocking buffer overnight at $4^{\circ} \mathrm{C}$. After washing three times in PBS $0.3 \%$ Triton X-100, they were incubated in relevant secondary antibodies conjugated to Alexa 488 and/or 594 (1:100; Molecular Probes, Eugene, OR) $1 \mathrm{hr}$ at room temperature (RT) in blocking buffer, and/or Alexa 594-conjugated phalloidin (Molecular Probes) at 1:30 in PBS for 5 min. All samples were examined at RT using a Radiance 2000 laser scanning confocal microscope (Bio-Rad, Hercules, CA) mounted on a Nikon Eclipse 200 modified laser safe microscope (Melville, NY) with an infinity-corrected $60 \times$ objective.

Software rendering. $Z$-series stacks were collected from astrocyte samples prepared and imaged as above, using $0.2 \mu \mathrm{m}$ on the $z$-axis between images. Stacks were then subjected to three-dimensional (3-D) rendering using Imaris software version 4.0 (Bitplane AG, Zurich, Switzerland).
Time lapse microscopy. Astrocyte cultures treated as described were imaged by Kohler illumination at $37^{\circ} \mathrm{C}$ using a Cooke Sensicam cooled CCD camera (Auburn Hills, MI) mounted on an Olympus (Tokyo, Japan) IX70 microscope with environmental chamber, using a $60 \times$ objective. Images were captured every $10 \mathrm{~min}$ using Scanalytics IPLab3.5 software (Fairfax, VA).

Migration assay. A wound was made in astrocyte cultures grown on glass confocal plates by scraping a soft sterile $200 \mu$ l pipette tip across the glass, and cultures were fixed and stained as described above at times shown. All dishes were imaged by immunofluorescence and Kohler illumination at RT with a $20 \times$ objective, using the CCD camera system described above. Fifty random $20 \times$ fields per culture condition were captured, and mean wound diameter was assessed in each field. Data were compared using unpaired $t$ test, with $p<0.05$ considered significant.

Bromodeoxyuridine labeling assay. Astrocyte cultures grown on glass confocal plates and treated with $10 \mathrm{ng} / \mathrm{ml} \mathrm{IL}-1 \beta$ for $24 \mathrm{hr}$ were subjected to wounding as above. At $4 \mathrm{hr}$ after wounding, $10 \mu \mathrm{M}$ bromodeoxyuridine (BrdU; Roche, Nutley, NJ) was added for $4 \mathrm{hr}$ to label mitotic cells, and cultures were then fixed 30 min with PBS $4 \%$ paraformaldehyde, rinsed two times in PBS, incubated in PBS and $2 \mathrm{M} \mathrm{HCl} / 0.25 \%$ Triton $\mathrm{X}-10020 \mathrm{~min}$, then neutralized in $0.1 \mathrm{M}$ sodium tetraborate, $\mathrm{pH}$ 8.5, for 10 min. After washing two times in PBS and once in PBS $0.1 \%$ glycine, they were blocked in PBS $0.3 \%$ Triton X-100 10\% goat serum 30 min, then incubated in anti-BrdU (1:200) in blocking buffer $2 \mathrm{hr}$ at RT. After washing three times in PBS and $0.3 \%$ Triton X-100, they were incubated in biotinylated secondary antibody (Vector Laboratories, Burlingame, CA) 1:200 in blocking buffer $30 \mathrm{~min}$ at RT, and then, after additional washing, in streptavidin-HRP (PerkinElmer, Boston, MA) 1:100 in blocking buffer for $30 \mathrm{~min}$. Reaction product was developed using diaminobenzidine (Sigma-Aldrich, St. Louis, MO), and cultures were imaged using the CCD camera system as above.

Rho $(\mathrm{G} 14 \mathrm{~V})$ transfection assay. A constitutively active (G14V) human RhoA expression construct was obtained from the Guthrie cDNA Resource Center (Sayre, PA), while the empty control construct pCDNA3.1 + was from Invitrogen (Carlsbad, CA). Astrocyte cultures were transfected with G14V Rho or empty vector using Lipofectamine Plus (Invitrogen) according to the manufacturer's instructions, and cotransfection with pCMV-DsRed-Express (Clontech, Palo Alto, CA) was used in some experiments to establish transfection efficiency. After $24 \mathrm{hr}$, half of the transfected cultures were treated with $10 \mathrm{ng} / \mathrm{ml} \mathrm{IL-1} \beta$, and after an additional $24 \mathrm{hr}$, all cultures were fixed and imaged with the CCD camera system described above, using a $20 \times$ objective. Twenty random fields per condition were captured, and each cell in all fields was assessed for stellation ( $\sim 600$ cells per condition). Data were compared using Mann-Whitney rank-sum test, with $p<0.05$ considered significant.

Rhotekin pulldown assay. Serum-starved astrocyte cultures treated as described were harvested in lysis buffer (25 mM HEPES, pH7.5, $150 \mathrm{~mm}$ $\mathrm{NaCl}, 1 \%$ Igepal CA-630, $10 \mathrm{~mm} \mathrm{MgCl}, 1 \mathrm{~mm}$ EDTA, and 2\% glycerol), and extracts were incubated $45 \mathrm{~min}$ at $4^{\circ} \mathrm{C}$ with GST-tagged rhotekin Rho-binding domain (RBD) bound to glutathione-agarose beads, as previously published (Ren et al., 1999). After extensive washing in lysis buffer, beads were resuspended in $2 \times$ SDS-PAGE loading buffer and boiled, then beads and supernatant were loaded onto $12.5 \%$ polyacrylamide gels and subjected to SDS-PAGE and Western blotting. Blots were probed for Rho (-A, -B, and -C).

Immunoblotting. SDS-PAGE and Western blotting were performed as previously described (Liu et al., 2000).

\section{Results}

\section{IL-1 $\beta$ induces dramatic changes in astrocyte phenotype}

To gain the best possible insight into effects of IL-1 $\beta$ on the structural phenotype of human astrocytes, we examined IL- $1 \beta$ treated and control cultures using a scanning electron microscope (Fig. 1.). After exposure to IL-1 $\beta$ (10 ng/ml), astrocytes exhibited dramatic structural changes, starting $\sim 6 \mathrm{hr}$ after the onset of treatment, and reaching completion at $\sim 24 \mathrm{hr}$. Treated cells developed a spherical cell body and multiple highly 
a.
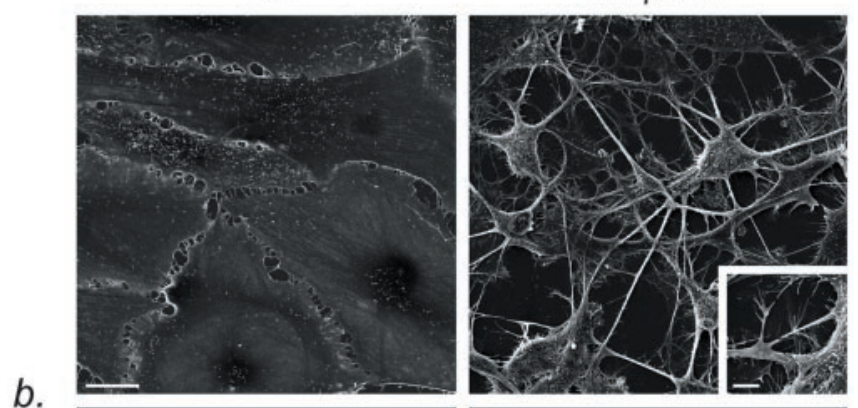

b.

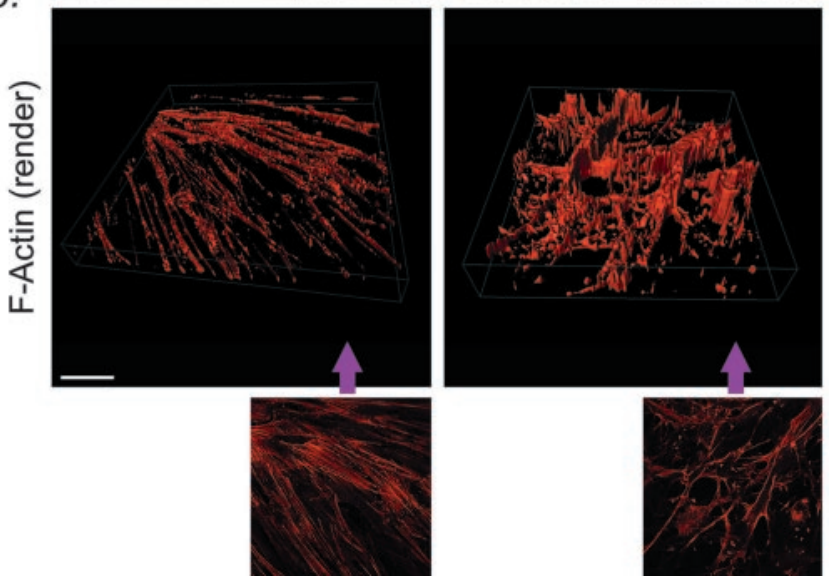

Figure 1. IL-1 $\beta$ induces dramatic changes in astrocyte phenotype. $a$, Primary human astro-

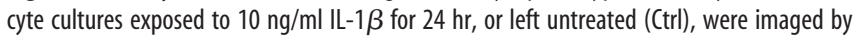
scanning electron microscopy. Scale bar, $20 \mu \mathrm{m}$. Treated cells developed a spherical cell body and multiple highly branched processes, terminating in prominent microvilli (inset; scale bar, 3 $\mu \mathrm{m})$. The experiment shown is representative of three experiments using astrocytes from three different brains. $b$, Confocal $z$-series of astrocytes treated as in $a$ and stained with phalloidin were subjected to software rendering to illustrate the arrangement of F-actin in these cultures. Individual images from the $z$-series stacks are shown directly below the renders. Scale bar, 40 $\mu \mathrm{m}$. The experiment illustrated is representative of three experiments using astrocytes from three different brains.

branched processes (Fig. 1a), terminating in long, prominent microvilli (Fig. 1a, inset), and under time-lapse microscopy these microvillar structures appeared highly labile, forming, extending, and collapsing on a minute-to-minute basis (data not shown). These changes occurred both in serum-starved cultures and in those grown in serum-containing medium, on polystyrene and on glass, and interestingly, they appeared to be irreversible: astrocytes did not revert to their former resting morphology following cytokine washout over a $7 \mathrm{~d}$ period.

To investigate the molecular changes underlying these structural effects, we used a combination of confocal imaging, 3-D rendering of confocal $z$-series, and immunoblotting. Using these techniques in combination allowed us to obtain a clear picture of both the spatial organization of specific molecules and an accurate measure of their overall levels within the cell. Using these methods, our first striking finding was that IL- $1 \beta$ induced a dramatic redistribution of the actin cytoskeleton in treated human astrocytes. Cells exposed to IL- $1 \beta$ showed complete loss of F-actin stress fibers, known to be a critical determinant of cellular morphology (Small et al., 1999) and relocalization of actin to the plasmalemma (Fig. 1b). Interestingly, immunoblotting experiments showed that this reorganization occurred in the absence of any change in overall actin levels within the cell (Fig. $2 d$ ).
IL-1 $\beta$ induces disruption of focal adhesions and deactivation of the contractile apparatus of the cell

Recruitment of fine actin bundles into stress fibers is typically accompanied by the formation of focal adhesions, which link the extracellular matrix to the cytoskeleton via heterodimeric integrin receptors and adapter proteins that include vinculin, talin, and paxillin (Wehrle-Haller and Imhof, 2002). They serve as sites for force transmission for the cell against the substratum, and also transmit signals into the cell via activation of FAK (Parsons et al., 2000 ). Because IL- $1 \beta$ induced reorganization of F-actin in astrocyte cultures, we determined whether focal adhesion structure and signaling were similarly altered. We found that these complexes were disrupted and their associated signaling function lost, in IL-1 $\beta$-treated astrocytes, with a time course that paralleled the redistribution of F-actin in these cells (Fig. 2). Resting astrocyte cultures exhibited large, prominent focal adhesions at the terminations of F-actin stress fibers that were strongly positive for vinculin (Fig. 2a) and for the activated signaling form of FAK (phosphorylated at Tyr576/577) (Fig. 2b). However, after treatment with $10 \mathrm{ng} / \mathrm{ml} \mathrm{IL-1} \beta$, vinculin underwent a dramatic redistribution, from a focal to a highly diffuse pattern (Fig. 2a,d), and FAK-P (Tyr576/577) was lost completely, as observed both by confocal imaging (Fig. $2 b$ ) and by immunoblotting (Fig. 2d). Interestingly, this dephosphorylation of FAK occurred in the absence of any alteration in overall FAK levels within the cell (Fig. $2 d$ ), and although the redistribution of vinculin that we observed was dramatic, its overall levels were likewise unchanged (Fig. 2d). Taken together, these data indicate that, as the actin cytoskeleton undergoes IL- $1 \beta$-mediated relaxation, focal adhesions lose both their structural integrity and their associated signaling role. Because FAK depends on the presence of coherent focal adhesion complexes for its activation state and signaling function (Parsons et al., 2000), we suggest that its deactivation is a logical consequence of disruption of these complexes.

The force transmitted by focal adhesions against the substratum of the cell is generated by contraction of the cellular actinmyosin stress fiber network, and myosin contractility also governs the formation and stability of stress fiber bundles and focal adhesion complexes (Chrzanowska-Wodnicka and Burridge, 1996). We therefore tested whether loss of these structures in IL- $1 \beta$-treated astrocytes was associated with loss of myosin contractility. Non-muscle myosin is known to be regulated by the phosphorylation and dephosphorylation of MLC: phosphorylation at Ser19 and Thr18 induces actin-activated myosin ATPase, leading to stress fiber formation, whereas dephosphorylation by myosin phosphatase deactivates myosin and allows cytoskeletal relaxation (Fukata et al., 2001). Using phosphospecific antibodies for MLC-P (Ser19) and (Thr18, Ser19), we found that IL-1 $\beta$ induced a striking deactivation of MLC in astrocyte cultures, with a time course that paralleled actin reorganization and focal adhesion disruption. Confocal imaging of resting astrocytes stained for MLC-P (Ser19) and F-actin showed MLC-P to be present in the astrocytic cytoplasm and associated with stress fibers (Fig. 2c), whereas imaging at higher magnification using a CCD camera system demonstrated the regular arrangement of individual phosphorylated MLC subunits in these cells (Fig. $2 f$ ). Conversely, confocal imaging of shape-changed astrocytes in IL- $1 \beta$-treated cultures showed loss of MLC-P (Fig. 2c), and this finding was confirmed using immunoblotting (Fig. $2 d$ ). IL-1 $\beta$-induced deactivation of MLC-P was observed at concentrations of the cytokine that were well within the recognized physiological range (Fig. 2e), and the same results were obtained using both anti-MLC (Ser19) and anti-MLC-P (Thr18, Ser19) (data not shown). Because deac- 
tivation of non-muscle myosin is known to be sufficient to induce cytoskeletal relaxation in several different cell types, these data suggest that IL- $1 \beta$-induced dephosphorylation of MLC may be a central event in the effects of this cytokine on astrocyte structure, leading to F-actin reorganization and associated disruption of focal adhesions. These data also indicate that the effects of IL- $1 \beta$ are associated with fundamental disruption of the contractile apparatus in human astrocytes and that this cytokine may inhibit both the generation of contractile force and its transmission against the surrounding substratum.

\section{IL-1 $\beta$ activates ERM adapter proteins, which localize to the microvillar compartment}

We next explored the molecular changes underlying the IL- $1 \beta$-induced development of prominent microvilli that we observed in our cultures (Fig. 3). A critical event in the formation of these structures is known to be phosphorylation of adapter proteins of the ERM family, at ezrin (Thr567), radixin (Thr564), or moesin (Thr558), allowing them to form a bridge between F-actin and integral membrane proteins at the plasmalemma (Mangeat et al., 1999). We therefore tested whether IL- $1 \beta$ regulated the phosphorylation state or overall levels of ERM family members in our human astrocyte cultures, using antibodies specific either for phospho- (ERMP), or for total ERMs. We found that treatment of astrocytes with IL- $1 \beta$ induced a rapid upregulation of ERM-P, without affecting overall ERM protein levels. This effect was observed using both confocal imaging (Fig. 3a) and immunoblotting (Fig. 3c), and was particularly apparent when confocal $z$-series were reconstituted using software rendering (Fig. $3 b$ ).

Activated ERM family members have been shown to associate with integral membrane proteins both directly [ICAM-1, ICAM-2, the sodium-hydrogen exchanger isoform 1 (NHE-1), CD43, and CD44] and indirectly via the scaffolding proteins EBP-50 and E3KARP (NHE-3, CFTR, and $\beta 2 \mathrm{AR}$ ) (for review, see Bretscher, 1999). Some of these proteins, such as ICAM-1, are known to be targets of IL- $1 \beta$ in human astrocytes (Lee et al., 1999). ICAM-1 plays an important role in leukocyte trafficking in the injured or inflamed CNS, and we therefore tested whether the effects of IL- $1 \beta$ on this adhesion molecule were coordinated in time and space with its effects on ERMs and F-actin in our astrocyte cultures. We found that the time course of IL- $1 \beta$-induced ERM phosphorylation paralleled that of induction of ICAM-1, as well as that of reorganization of F-actin to the plasmalemma (Fig. $3 c$ ), and that in addition, ERM-P and ICAM-1 both localized solely to microvilli in IL- $1 \beta$-treated astrocytes (Fig. $3 d$ ). Taken together, these findings suggest that coordinated effects of IL- $1 \beta$ on actin, ERMs and ICAM-1 may all be involved in the formation of ICAM-1-bearing microvilli by reactive astrocytes in the injured human CNS.
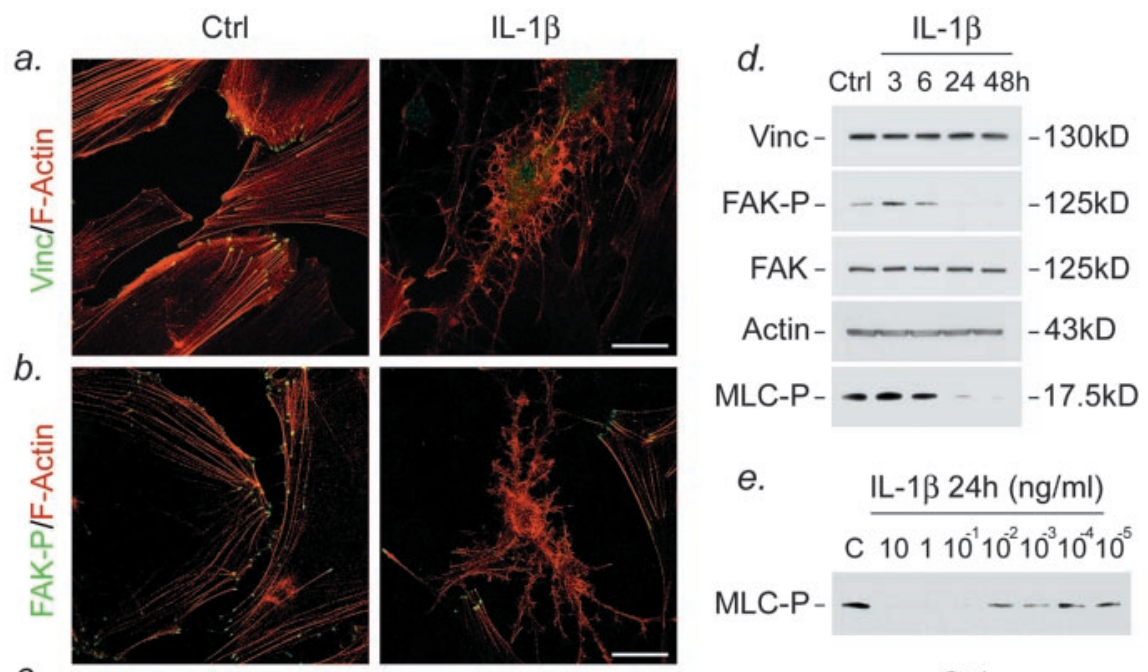

$f$.
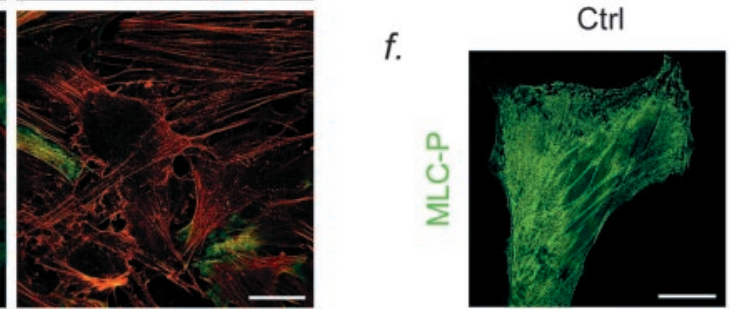

Figure 2. IL-1 $\beta$ induces disruption of focal adhesions and deactivation of the contractile apparatus of the cell. $a-c$ are confocal L-1 $\beta$ for times shown, or left untreated. Blots were probed with antibodies specific for vinculin, phospho-focal adhesion kinase (5) total focal adhesion kinase, actin, and phospho-myosin light chain 2 (Ser19). The experiment shown is represenIL-1 $\beta$. f, High-power CCD image of a single lamellipodium of an untreated primary human astrocyte immunostained for phosphomyosin light chain 2 (Ser19). Scale bar, $10 \mu \mathrm{m}$.

\section{IL-1 $\beta$-treated astrocytes show restricted migratory capacity}

Because our data showed that IL- $1 \beta$ appeared to reorganize, disrupt, or deactivate the actin cytoskeleton, focal adhesions, and nonmuscle myosins in astrocytes, we wondered what the functional consequences of these changes might be. All of these structures are known to be critical contributors to cellular motility and migration (Small et al., 1999; Smilenov et al., 1999), and we therefore tested whether the migratory capacity of astrocytes was altered in IL- $1 \beta$-treated cultures. Briefly, we stimulated astrocyte migration in a monolayer using an in vitro scratch-wound assay, as previously described by Etienne-Manneville and Hall (2001), and then compared the resulting migration in IL- $1 \beta$-treated cultures versus matched controls (Fig. 4). Initial wound diameter was very similar for both conditions $(\sim 500 \mu \mathrm{m})$, and astrocytes in control cultures closed this gap in $\sim 16 \mathrm{hr}$, becoming fully confluent by $24 \mathrm{hr}$ (Fig. 4a,b). Conversely, wound closure was strikingly delayed in IL$1 \beta$-treated cultures (Fig. $4 a$ ), with wound diameter being significantly greater in these cultures than in controls at both 16 and $24 \mathrm{hr}$ ( $p<0.0001$ for both time points) (Fig. $4 b$ ).

To understand better the mechanism underlying this delay, we used time-lapse microscopy, which revealed interesting differences in the movement characteristics of IL- $1 \beta$-treated astrocytes versus controls. In control cultures, migrating cells moved in a coordinated manner, first extending lamellipodia and establishing contacts, then contracting to pull themselves forward. In 

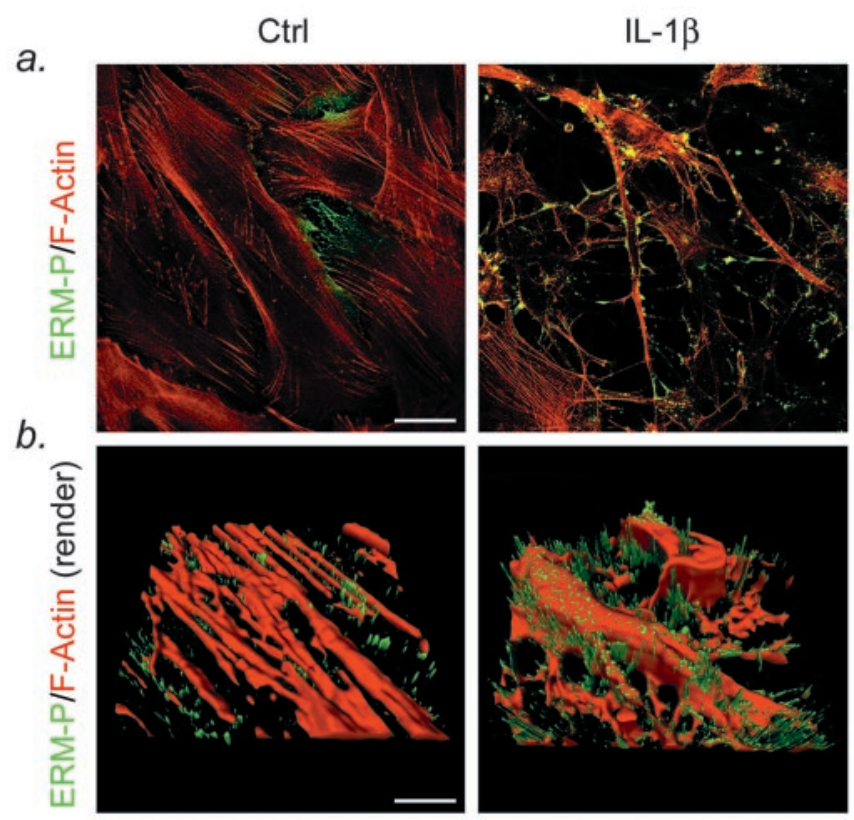

C. $d$.
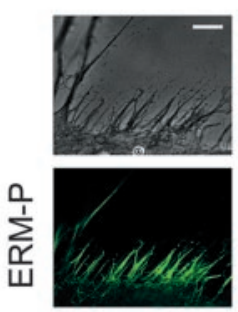

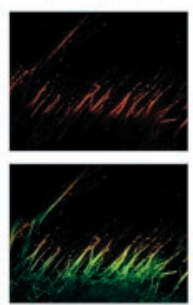

ICAM-1

Figure 3. IL-1 $\beta$ activates ERM adapter proteins, which localize to the microvillar compartment. a shows confocal images of astrocytes treated with IL-1 $\beta$ as in Figure 1, or left untreated (Ctrl), and stained with phalloidin (red) and an antibody specific for phospho-ezrin (Thr567)/ radixin (Thr564)/moesin (Thr558) (green). The experiment shown is representative of three experiments using astrocytes from three different brains. Scale bar, $30 \mu \mathrm{m}$. $b$, Confocal $z$-series of astrocytes treated and stained as in $a$ were subjected to software rendering. Scale bar, 20 $\mu \mathrm{m}$. c, Western blots of total cell homogenates treated as shown were probed with antibodies specific for VCAM-1, ICAM-1, phospho-ezrin(Thr567)/radixin(Thr564)/moesin(Thr558), and total ezrin/radixin/moesin. The experiment illustrated is representative of three experiments using astrocytes from three different brains. $d$, High-power $C C D$ images of microvilli on the surface of astrocytes treated with IL-1 $\beta$ as in ( $a$ ) and double-stained with antibodies specific for ICAM-1 (red) and phospho-ezrin(Thr567)/radixin(Thr564)/moesin(Thr558) (green). Red channel, green channel, merged, and Kohler images are shown. Scale bar, $4 \mu \mathrm{m}$.

contrast, Il-1 $\beta$-treated astrocytes appeared to extend and collapse processes at random, and the motility that we observed in these cultures appeared uncoordinated. This would suggest that the differences that we observed in wound healing resulted predominantly from an alteration in the movement characteristics of cytokine-treated versus control cultures, rather than from differences in mitotic activity at the wound edge. This conclusion was supported by the results of BrdU labeling experiments, which did not demonstrate BrdU labeling focused at the wound edge in either control or IL-1 $\beta$-treated astrocyte cultures (data not shown).

\section{IL-1 $\beta$ regulates astrocytic phenotype via deactivation of} Rho GTPases

We next addressed the mechanism underlying the structural effects of IL-1 $\beta$. F-actin stress fibers, focal adhesions, MLC, and a.
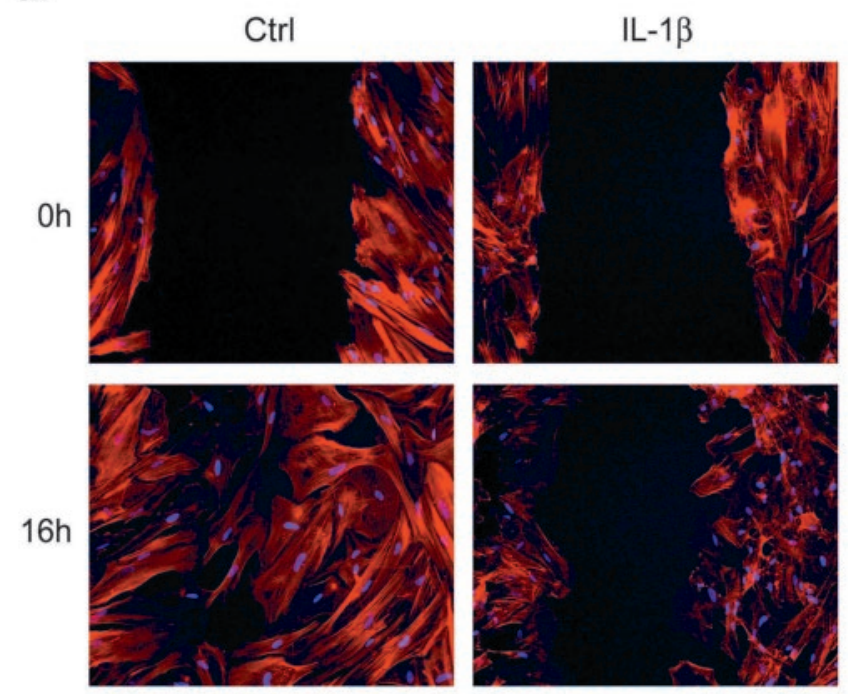

$24 \mathrm{~h}$
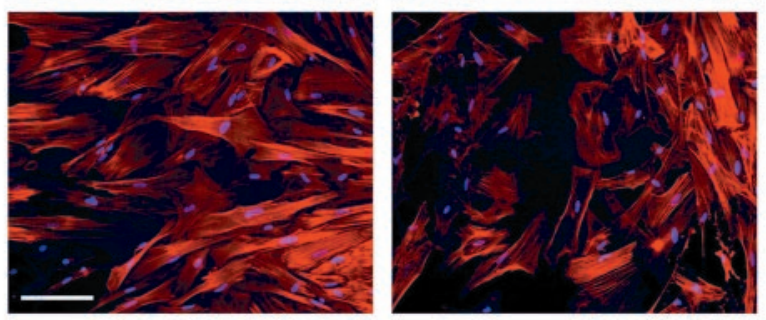

b.

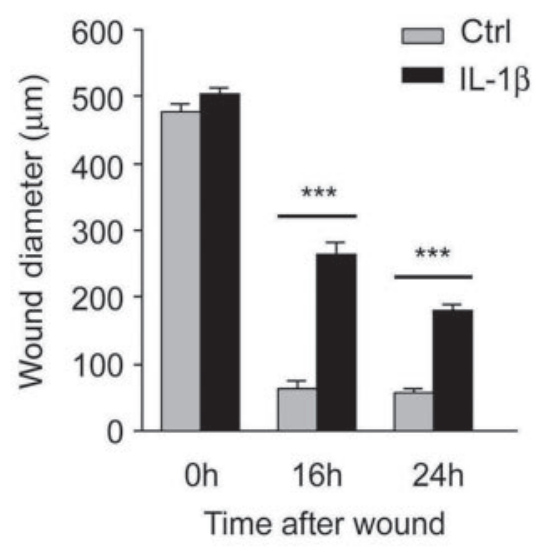

$* * *=P<0.0001$

Figure 4. IL-1 $\beta$-treated astrocytes show restricted migratory capacity. a, Astrocyte cultures were grown to confluence and treated with $10 \mathrm{ng} / \mathrm{ml} \mathrm{IL-1 \beta}$ for $24 \mathrm{hr}$. A wound was then made in treated and control cultures, and cells were fixed and stained with phalloidin (red) and DAPI (blue) at times shown. Scale bar, $150 \mu \mathrm{m}$. b, Quantitation of wound diameter was performed, and data were subjected to statistical analysis as described in Materials and Methods. Data shown is from a single experiment using triplicate samples from the same brain and is representative of five independent experiments using astrocytes from five different brains.

ERM proteins have all previously been identified as targets of a single regulatory mechanism, a signaling pathway mediated by small GTPases of the Rho subfamily, and their downstream effector ROCK (for review, see Amano et al., 2000). This pathway is known to be one of the master regulators of cell shape, motility, and migration (Schmitz et al., 2000; Riento and Ridley, 2003), and we therefore tested whether the effects that we observed re- 
sulted from intersection of IL- $1 \beta$ signaling with the Rho-ROCK signaling pathway (Fig. 5). First, we compared the results of IL- $1 \beta$ treatment with effects produced by ROCK inhibitors in our human astrocyte cultures. Strengthening our hypothesis that the two pathways may intersect, we found that blockade of ROCK using the selective ROCK inhibitors Y-27632 or HA1077 induced structural and molecular changes similar to those observed in the wake of IL- $1 \beta$ treatment (Fig. $5 a-e$ ). Astrocytes treated with these inhibitors developed a spherical cell body and multiple highly branched processes (Fig. 5a), although they lacked the prominent microvilli that were observed in IL- $1 \beta$ treated cultures (Fig. $5 a$, inset). In addition, these morphological changes were temporary, in contrast to the time course of the effects of IL- $1 \beta$ : the changes were complete by $6 \mathrm{hr}$ after treatment with $10 \mu \mathrm{M}$ Y-27632 and had reversed by $48 \mathrm{hr}$. The alterations in morphology were associated with loss of F-actin stress fibers (Fig. $5 b, c$ ), disruption of focal adhesions as illustrated by diffuse redistribution of vinculin (Fig. 5b) and dephosphorylation of FAK (Fig. $5 d$ ), and loss of myosin contractility as evidenced by dephosphorylation of MLC (Fig. $5 c, d$ ). Interestingly, FAK dephosphorylation induced by ROCK inhibitors appeared temporary, whereas dephosphorylation of MLC was more persistent (Fig. $5 d$ ). Taken together, these data indicate that F-actin, focal adhesions, and nonmuscle myosin are all regulated, directly or indirectly, both by IL- $1 \beta$ and by the Rho-ROCK pathway in human astrocytes. However, in contrast to its effects on MLC and FAK, ROCK blockade had no effect on ERM phosphorylation (data not shown), a result that correlated with the lack of prominent microvilli in Y27632treated cultures. This may suggest either that the phosphorylation status of ERM proteins is not ROCK-dependent in human astrocytes or that the influence of another pathway on ERMs outweighs that of the Rho-ROCK pathway in this cell type.

We next tested whether more upstream targets of the Rho-ROCK axis were regulated by IL- $1 \beta$ in our cultures. The activation state of non-muscle myosin is known to depend on a balance between phosphorylation of MLC at Ser19 and Thr18 by ROCK, and its dephosphorylation by myosin phosphatase (Fig. 6). ROCK also deactivates myosin phosphatase via phosphorylation of its targeting subunit (MYPT) at Thr696, thereby further enhancing MLC phosphorylation (Feng et al., 1999). Using a phosphospecific antibody, we found that there was progressive loss of MYPT-P (Thr696) in IL-1 $\beta$-treated astrocyte cultures, with a time course that paralleled the effects of this cytokines on MLC (Fig. $5 e$ ).
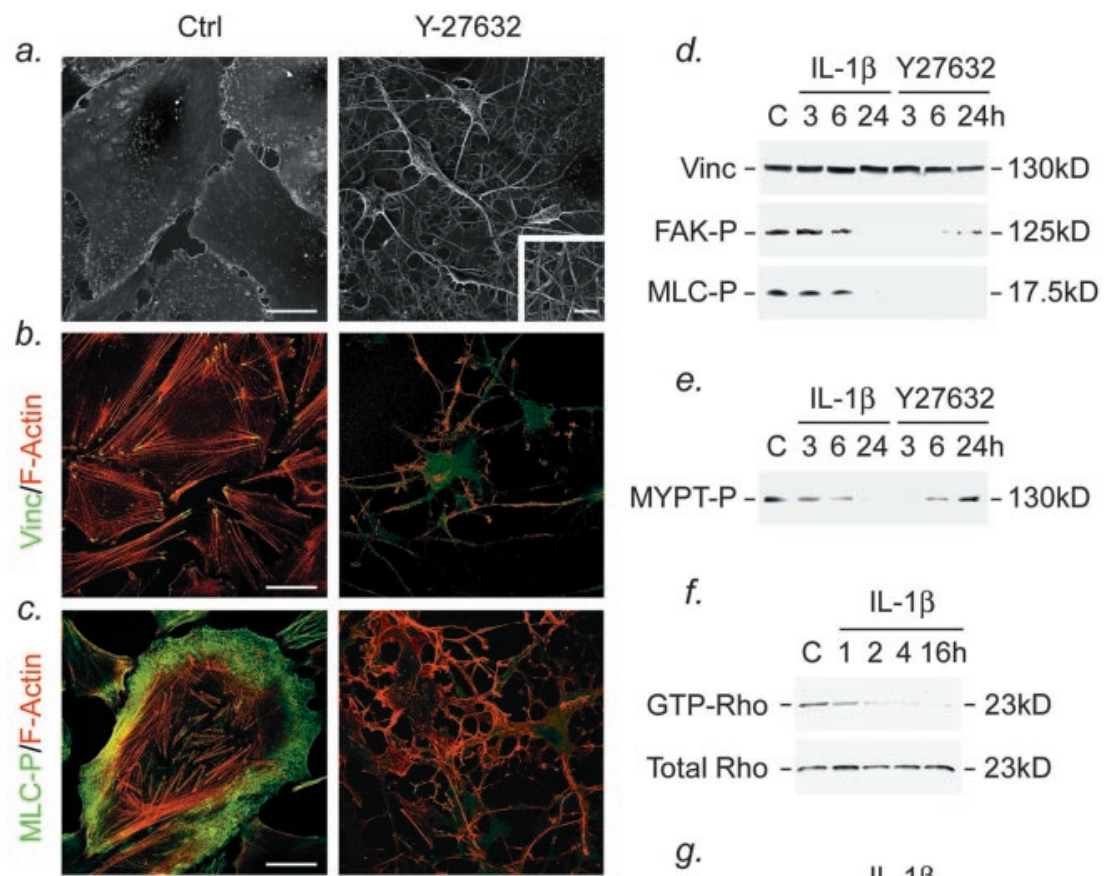

e.

IL-1及 Y27632

C $\overline{3624} \overline{3624} \mathrm{~h}$

MYPT-P - - - - $-130 \mathrm{kD}$

$f$.

$$
\text { C } \frac{\mathrm{IL}-1 \beta}{12416 \mathrm{~h}}
$$

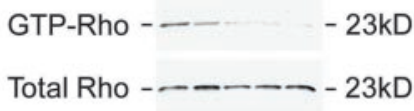

g.

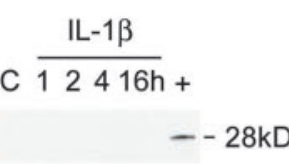

Figure 5. IL-1 $\beta$ regulates astrocytic phenotype via deactivation of Rho GTPases. $a$, Astrocyte cultures treated with the ROCK inhibitor Y-27632 $10 \mu \mathrm{m}$ for $24 \mathrm{hr}$, or left untreated (Ctrl), were imaged by scanning electron microscopy. Scale bar, $25 \mu \mathrm{m}$. Cells treated with Y-27632 developed a spherical cell body and multiple highly branched processes, but did not develop prominent microvilli (inset; scale bar, $5 \mu \mathrm{m}$ ). Data shown are representative of three experiments using astrocytes from three different brains. $b$ and c are confocal images of astrocytes treated as in $a$, stained with phalloidin (red) and antibodies (green) specific for vinculin $(b)$, or phospho-myosin light chain $2(\operatorname{Ser} 19)(c)$, and imaged by confocal microscopy. Results illustrated are representative of three independent experiments using astrocytes from three different brains. Scale bars, $40 \mu \mathrm{m}$. $d$ and e show immunoblots comparing levels of vinculin, phospho-focal adhesion kinase (Tyr576/577), phospho-myosin light chain 2 (Ser19) (d) and phospho-myosin phosphatase targeting subunit (Thr696) (e) in total lysates from astrocyte cultures treated with $10 \mathrm{ng} / \mathrm{ml} \mathrm{IL-1 \beta}$ or $10 \mu \mathrm{m}$ Y-27632 for times shown. The experiment illustrated is representative of three experiments using astrocytes from three different brains in all cases. $f$, Astrocyte cultures treated with $10 \mathrm{ng} / \mathrm{ml} \mathrm{IL-1} \beta$ for times shown were subjected to pulldown assay with rhotekin RBD, which binds the active form of Rho GTPase. Levels of total Rho in the same samples were also assessed in parallel (bottom blot). The experiment illustrated is representative of three independent experiments using astrocytes from three separate brains. $g$, Astrocytes were treated with IL-1 $\beta$ for times shown, or with EGF (positive control, lane marked + ), and blots of total cell lysates were probed with an antibody specific for phospho-Rac1/cdc42(Ser71). Data shown is representative of three independent experiments using astrocytes from three different brains. $h$ compares the effect of IL- $1 \beta$ on astrocytic morphology in cultures transfected with constitutively active Rho (G14V) versus empty vector-transfected controls. Constitutively active Rho significantly inhibited the effects of IL-1 $\beta$ on astrocytic morphology $(p<0.0001)$. Data shown is from an individual experiment using triplicate samples from the same brain for each condition and is representative of three independent experiments using astrocytes from three different brains.

Loss of MYPT-P (Thr696) was also observed after treatment of cultures with ROCK inhibitors, confirming its regulation by both IL- $1 \beta$ and by the Rho-ROCK pathway in human astrocytes (Fig. 5e). The effect of ROCK inhibitors was rapid and temporary, whereas that of IL- $1 \beta$ was long-lasting, as we had already observed with several of our other findings in this study. 


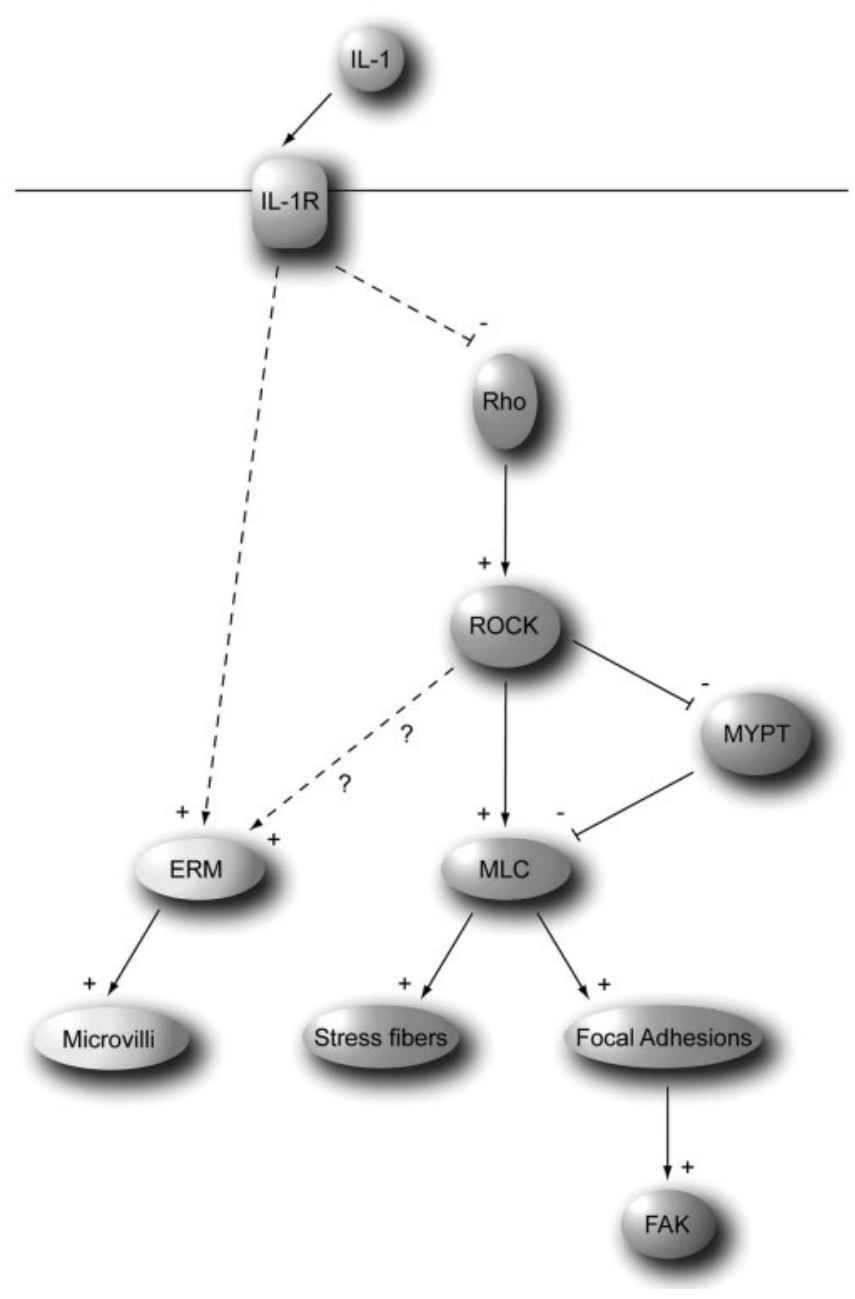

Figure 6. Diagrammatic representation of the interaction of IL-1 $\beta$ with the Rho-ROCK signaling axis. IL-1 $\beta$ acts to deactivate Rho, leading in turn to dephosphorylation of MYPT and MLC, loss of stress fibers, and disruption of focal adhesions and dephosphorylation of FAK. Conversely, IL-1 $\beta$ upregulates phosphorylation of ERM family members.

Next, we used a more direct approach to investigate a potential link between IL-1 $\beta$ and Rho (Fig. $5 f$ ). We compared the levels of activated (GTP-) Rho in lysates from IL- $1 \beta$-treated and control astrocyte cultures, using a rhotekin Rho-binding domain (RBD) pulldown technique that has been published previously (Ren et al., 1999). Rhotekin RBD binds specifically to activated (GTP-) Rho but not to inactive (GDP-) Rho. Briefly, we incubated lysates from cytokine-treated and control astrocyte cultures with RBD-conjugated agarose beads, washed the beads, and then compared the levels of bound Rho by immunoblotting. Levels of total Rho in the same samples were also assessed in parallel. We found that, as the results of our inhibitor experiments had suggested, IL- $1 \beta$ treatment of human astrocytes led to Rho deactivation, with a time course that was compatible with the other structural effects of this cytokine. After exposure of astrocytes to IL-1 $\beta$ (10 ng/ml), levels of activated Rho declined steadily, with very little activated Rho detected by $16 \mathrm{hr}$ after treatment onset (Fig. $5 f$ ). Although IL- $1 \beta$ induced Rho deactivation, it had no effect on the overall levels of total Rho in these cultures (Fig. 5f). The effect on Rho activation also appeared to be specific: we found that IL- $1 \beta$ had no effect on the activation state of two other small GTPases, Rac and cdc42, in our cultures, as assessed by immunoblotting using an antibody specific for their activated forms (Fig. $5 g$ ).

Finally, we performed experiments to determine whether IL$1 \beta$-mediated deactivation of Rho was sufficient to account for the structural effects of this cytokine. We used a transfection paradigm to introduce a constitutively active (G14V) form of Rho, or the relevant empty vector control, into matched astrocyte cultures, and then treated the cells with $10 \mathrm{ng} / \mathrm{ml} \mathrm{IL-1 \beta}$. We found that an IL- $1 \beta$-induced change in astrocytic morphology was significantly inhibited in cultures transfected with constitutively active Rho $(p<0.0001)$ (Fig. 5h), whereas the same construct had no significant effect on morphology in untreated cultures, as expected. These data demonstrate that constitutively active Rho is sufficient to rescue astrocytes from the structural effects of IL- $1 \beta$, indicating that the deactivation of Rho GTPases by this cytokine is a critical event in its regulation of astrocyte morphology.

\section{Discussion}

A combination of studies, performed in vivo and in vitro by multiple groups, has convincingly implicated a number of cytokines in the generation or modulation of an astrocytic scar. Cytokines for which the evidence of direct involvement is most compelling include IL- $1 \beta, \mathrm{TNF} \alpha$, IFN $\gamma$, and TGF $\beta$, with each fulfilling a different and specific function, inducing its own characteristic transcriptional signature (John et al., 2002). Based on available evidence, the specific role of IL- $1 \beta$ appears to be the primary initiation of an astrocytic injury response. For example, injection of IL- $1 \beta$ is sufficient to induce a reactive astrogliosis in the mammalian brain (Giulian et al., 1988), whereas the astrocytic scar that would normally occur after CNS injury fails to form in animals deficient for this cytokine (Herx and Yong, 2001). In addition, the transcriptional profile induced by IL- $1 \beta$ in primary cultures of human astrocytes is overwhelmingly proinflammatory and includes multiple genes known to be involved in the earliest stages of the injury response (John et al., 2002). IL-1 $\beta$ also induces structural changes in astrocytes in vitro that are reminiscent of those observed in reactive astrocytes in vivo (Liu et al., 1994), and to date it is the only cytokine known to have this effect (Lee and Brosnan, 1997). Interestingly, however, although IL-1 $\beta$ is known to be a potent regulator of gene transcription, exerting its effects via activation of transcription factors including NF- $\kappa \mathrm{B}$ and AP-1 (Ghosh and Karin, 2002; O'Neill, 2002), the mechanism underlying its structural effects has remained unresolved (Liu et al., 1994; Safavi-Abbasi et al., 2001).

In the current study, we set out to identify the molecular events underlying the structural effects of IL- $1 \beta$ in human astrocytes and the signaling mechanism responsible. Our first striking finding was that IL- $1 \beta$ caused a dramatic reorganization of the actin cytoskeleton, inducing loss of stress fibers and relocalization of F-actin to the plasmalemma. The actin cytoskeleton consists of an integrated array of interconnected filament-assemblies, of which stress fibers, lamellipodia and filopodia are the most cited (Hall, 1998). In contrast to lamellipodia and filopodia, which are protrusive elements consisting of unipolar actin filaments, stress fibers contain bipolar arrays of actin and myosin type II, and thus possess the ability to contract and exert tension. Unlike lamellipodia and filopodia, they therefore require substrate anchorage for their formation (Small et al., 1999).

Stress fibers are typically anchored by focal adhesion complexes that serve as sites both for signaling and for force transmission against the substratum, with the force being generated by contraction of the cellular actin-myosin stress fiber network. Myosin contractility, controlled by phosphorylation of MLC, 
also governs the formation and stability of stress fiber bundles and focal adhesion complexes (Chrzanowska-Wodnicka and Burridge, 1996). We found that IL- $1 \beta$-induced reorganization of actin was accompanied by disruption of focal adhesion complexes and loss of their signaling function, and that these changes were further associated with deactivation of non-muscle myosin, as assessed by immunoblotting for MLC-P. Taken together, these data indicate that IL- $1 \beta$ induces a fundamental disruption of the contractile apparatus in human astrocytes, inhibiting both the generation and the transmission of contractile force.

Available evidence implicates one pathway more than any other in the control of myosin contractility and stress fiber and focal adhesion formation. In mammalian cells, small GTPases of the Rho family (part of the Ras superfamily) have been shown to act as central regulators of cell morphology, contractility, and migration, with complementary aspects of these processes being handled by the cdc42, Rac, and Rho subfamilies (EtienneManneville and Hall, 2002). For example, Rac induces formation of lamellipodia (Ridley et al., 1992; Machesky and Hall, 1997), whereas Rho controls stress fiber and focal adhesion assembly (Ridley and Hall, 1992) via activation of myosin contractility (Chrzanowska-Wodnicka and Burridge, 1996). A large proportion of the signals from Rho are handled by ROCK, which regulates the activity of specific downstream effectors, the best characterized of which is MLC (Amano et al., 1997). ROCK controls MLC activation both via direct phosphorylation, and indirectly via the inactivation of myosin phosphatase through the phosphorylation of its targeting subunit (MYPT), thus controlling myosin contraction and stress fiber and focal adhesion formation in nonmuscle cells (Amano et al., 2000). The Rho-ROCK-MLC axis thus appears to be the critical controller of these processes in mammalian systems, in turn suggesting the potential for the involvement of this pathway in the effects of IL- $1 \beta$ on astrocyte structure. Interactions between cytokine signaling and small GTPases, including Rho, have been reported in fibroblasts, HeLa cells, and neurons (Puls et al., 1999; Singh et al., 1999, Neumann et al., 2002), but such interactions have not previously been implicated in the generation of a reactive astrogliosis.

Other downstream molecules have also been proposed as targets of the Rho-ROCK axis, including LIM kinase (Maekawa et al., 1999), adducin, and ERM microvillar adapter proteins (Matsui et al., 1998), although the role of ROCK in ERM phosphorylation has more recently been called into question (Matsui et al., 1999). We found that IL- $1 \beta$ had opposing effects on MLC and ERMs in our experiments, and so we used pharmacological inhibitors to elucidate the role of ROCK in the regulation of these molecules in our astrocyte cultures. Whereas astrocytes treated with these inhibitors exhibited dephosphorylation of MYPT and MLC, we observed no effect on the phosphorylation state of ERM proteins. In the context of the current study, these data would indicate that, although both IL- $1 \beta$ and ROCK regulate MYPT and MLC, ERMs are regulated by IL- $1 \beta$ but not by ROCK. This in turn suggested the potential for the effects of IL- $1 \beta$ on MLC, actin, and focal adhesions to be mediated via inhibition of the Rho-ROCK pathway, and we confirmed that IL- $1 \beta$ deactivated Rho using pulldown assays with the Rho-binding domain of rhotekin. We further demonstrated that a constitutively active Rho construct rescued astrocytes from developing an IL- $1 \beta$-induced reactive phenotype, indicating that the effect of IL- $1 \beta$ on Rho activation is central to the structural effects of this cytokine in primary human astrocytes.

Because our data demonstrated that IL- $1 \beta$ treatment led to disruption of the contractile apparatus in primary human astro- cytes, we investigated the functional consequences of these effects. The results of these experiments showed that IL- $1 \beta$-treated astrocytes possess a restricted migratory capacity, and further studies using time-lapse microscopy and BrdU labeling suggested that this was associated with uncoordinated astrocyte motility in IL- $1 \beta$-treated cultures. Migration requires coordinated lamellipodium extension coupled with the development of new cell adhesions to the extracellular substrate, followed by cell body contraction and tail detachment (Lauffenburger and Horwitz, 1996). Because this process is believed to be dependent on the synchronized activities of different small GTPases (Etienne-Manneville and Hall, 2002), an alteration in the activation state of any of these GTPases might be expected to disrupt the migration process. For example, deactivation of Rho might be expected to result in cells capable of extending lamellipodia and filopodia, but incapable of contraction, and hence unable to migrate or maintain a coordinated morphology, and this corresponds well to the phenotype that we observed in our IL- $1 \beta$-treated astrocyte cultures.

Interestingly, our data provide a counterpoint to the effect of TNF $\alpha$ or trauma on the activation state of Rho GTPases in neurons (Neumann et al., 2002; Dubreuil et al., 2003). Our results suggest that the initiating signal of a reactive astrogliosis is associated not with activation of Rho GTPases, but, conversely, with their deactivation. That the response of astrocytes differs from that found in neurons is perhaps not surprising, because the persistence of ramified astrocytes at sites where neuronal processes are lost is the hallmark of essentially all chronic injury in the CNS. Our data showing IL- $1 \beta$-mediated dephosphorylation of MLC and MYPT provide strong support for our results on Rho, because the phosphorylation states of these molecules are known to be exquisitely sensitive to the level of activation of the RhoROCK pathway.

In summary, we have shown in these experiments that IL- $1 \beta$ signaling regulates the phenotype of astrocytes via an interaction with a pathway mediated by small GTPases of the Rho subfamily, and their downstream effector ROCK (Fig. 6). This pathway regulates the morphology, contractility, and migration of mammalian cells via effects on F-actin and its interactions with focal adhesions and non-muscle myosins, and our data demonstrate that IL- $1 \beta$ regulates all of these events in human astrocytes, and furthermore, that its effects are mimicked by blockade of ROCK. We have also demonstrated directly that IL- $1 \beta$ deactivates Rho and that a constitutively active Rho construct rescues astrocytes from developing an IL- $1 \beta$-induced reactive phenotype. These data implicate cytokine regulation of the Rho-ROCK pathway in the genesis of a reactive astrogliosis, and on the basis of our data, we predict that targeted intervention at the level of this pathway may allow manipulation of the astrocytic scar in inflammatory diseases of the human CNS.

\section{References}

Amano M, Chihara K, Kimura K, Fukata Y, Nakamura N, Matsuura Y, Kaibuchi K (1997) Formation of actin stress fibers and focal adhesions enhanced by Rho-kinase. Science 275:1308-1311.

Amano M, Fukata Y, Kaibuchi K (2000) Regulation and functions of Rhoassociated kinase. Exp Cell Res 261:44-51.

Bretscher A (1999) Regulation of cortical structure by the ezrin-radixinmoesin protein family. Curr Opin Cell Biol 11:109-116.

Bush TG, Puvanachandra N, Horner CH, Polito A, Ostenfeld T, Svendsen CN, Mucke L, Johnson MH, Sofroniew MV (1999) Leukocyte infiltration, neuronal degeneration, and neurite outgrowth after ablation of scarforming, reactive astrocytes in adult transgenic mice. Neuron 23:297-308.

Chrzanowska-Wodnicka M, Burridge K (1996) Rho-stimulated contractility drives the formation of stress fibers and focal adhesions. J Cell Biol 133:1403-1415. 
Danbolt NC (2001) Glutamate uptake. Prog Neurobiol 65:1-105.

Delhase M, Hayakawa M, Chen Y, Karin M (1999) Positive and negative regulation of IkappaB kinase activity through IKKbeta subunit phosphorylation. Science 284:309-313.

Dubreuil CI, Winton MJ, McKerracher L (2003) Rho activation patterns after spinal cord injury and the role of activated Rho in apoptosis in the central nervous system. J Cell Biol 162:233-243.

Etienne-Manneville S, Hall A (2001) Integrin-mediated activation of Cdc42 controls cell polarity in migrating astrocytes through PKCzeta. Cell 106:489-498.

Etienne-Manneville S, Hall A (2002) Rho GTPases in cell biology. Nature 420:629-635

Feng J, Ito M, Ichikawa K, Isaka N, Nishikawa M, Hartshorne DJ, Nakano T (1999) Inhibitory phosphorylation site for Rho-associated kinase on smooth muscle myosin phosphatase. J Biol Chem 274:37385-37390.

Fields RD, Stevens-Graham B (2002) New insights into neuron-glia communication. Science 298:556-562.

Fukata Y, Amano M, Kaibuchi K (2001) Rho-Rho-kinase pathway in smooth muscle contraction and cytoskeletal reorganization of nonmuscle cells. Trends Pharmacol Sci 22:32-39.

Ghosh S, Karin M (2002) Missing pieces in the NF-kappaB puzzle. Cell 109:S81-S96.

Giulian D, Woodward J, Young DG, Krebs JF, Lachman LB (1988) Interleukin-1 injected into mammalian brain stimulates astrogliosis and neovascularization. J Neurosci 8:2485-490.

Hall A (1998) Rho GTPases and the actin cytoskeleton. Science 279:509-514.

Herx LM, Yong VW (2001) Interleukin-1 beta is required for the early evolution of reactive astrogliosis following CNS lesion. J Neuropathol Exp Neurol 60:961-971.

Janzer RC, Raff MC (1987) Astrocytes induce blood-brain barrier properties in endothelial cells. Nature 325:253-257.

John GR, Scemes E, Suadicani SO, Liu JS, Charles PC, Lee SC, Spray DC, Brosnan CF (1999) IL-1beta differentially regulates calcium wave propagation between primary human fetal astrocytes via pathways involving P2 receptors and gap junction channels. Proc Natl Acad Sci USA 96:11613-11618.

John GR, Shankar SL, Shafit-Zagardo B, Massimi A, Lee SC, Raine CS, Brosnan CF (2002) Multiple sclerosis: re-expression of a developmental pathway that restricts oligodendrocyte maturation. Nat Med 8:1115-1121.

Lauffenburger DA, Horwitz AF (1996) Cell migration: a physically integrated molecular process. Cell 84:359-369.

Lee SC, Brosnan CF (1997) Molecular biology of glia: astrocytes. In: Molecular biology of multiple sclerosis, (Russell WC, ed), pp 71-96. New York: Wiley.

Lee SJ, Park JY, Hou J, Benveniste EN (1999) Transcriptional regulation of the intercellular adhesion molecule- 1 gene by proinflammatory cytokines in human astrocytes. Glia 25:21-32.

Liu J, Zhao ML, Brosnan CF, Lee SC (1996) Expression of type II nitric oxide synthase in primary human astrocytes and microglia: role of IL-1beta and IL-1 receptor antagonist. J Immunol 157:3569-3576.

Liu JS, John GR, Sikora A, Lee SC, Brosnan CF (2000) Modulation of interleukin-1beta and tumor necrosis factor alpha signaling by P2 purinergic receptors in human fetal astrocytes. J Neurosci 20:5292-5299.

Liu W, Shafit-Zagardo B, Aquino DA, Zhao ML, Dickson DW, Brosnan CF, Lee SC (1994) Cytoskeletal alterations in human fetal astrocytes induced by interleukin-1 beta. J Neurochem 63:1625-1634.

Machesky LM, Hall A (1997) Role of actin polymerization and adhesion to extracellular matrix in Rac- and Rho-induced cytoskeletal reorganization. J Cell Biol 138:913-926.

Maekawa M, Ishizaki T, Boku S, Watanabe N, Fujita A, Iwamatsu A, Obinata T, Ohashi K, Mizuno K, Narumiya S (1999) Signaling from Rho to the actin cytoskeleton through protein kinases ROCK and LIM-kinase. Science 285:895-898.
Mangeat P, Roy C, Martin M (1999) ERM proteins in cell adhesion and membrane dynamics. Trends Cell Biol 9:187-192.

Matsui T, Maeda M, Doi Y, Yonemura S, Amano M, Kaibuchi K, Tsukita S Tsukita S (1998) Rho-kinase phosphorylates COOH-terminal threonines of ezrin/radixin/moesin (ERM) proteins and regulates their headto-tail association. J Cell Biol 140:647-657.

Matsui T, Yonemura S, Tsukita S, Tsukita S (1999) Activation of ERM proteins in vivo by Rho involves phosphatidyl-inositol 4-phosphate 5-kinase and not ROCK kinases. Curr Biol 9:1259-1262.

Neumann H, Schweigreiter R, Yamashita T, Rosenkranz K, Wekerle H, Barde YA (2002) Tumor necrosis factor inhibits neurite outgrowth and branching of hippocampal neurons by a rho-dependent mechanism. J Neurosci 22:854-862.

O'Neill LA (2002) Signal transduction pathways activated by the IL-1 receptor/toll-like receptor superfamily. Curr Top Microbiol Immunol 270:47-61.

Parsons JT, Martin KH, Slack JK, Taylor JM, Weed SA (2000) Focal adhesion kinase: a regulator of focal adhesion dynamics and cell movement. Oncogene 19:5606-5613.

Puls A, Eliopoulos AG, Nobes CD, Bridges T, Young LS, Hall A (1999) Activation of the small GTPase Cdc42 by the inflammatory cytokines TNF(alpha) and IL-1, and by the Epstein-Barr virus transforming protein LMP1. J Cell Sci 112:2983-2992.

Ren XD, Kiosses WB, Schwartz MA (1999) Regulation of the small GTPbinding protein Rho by cell adhesion and the cytoskeleton. EMBO $18: 578-585$.

Ridley AJ, Hall A (1992) The small GTP-binding protein rho regulates the assembly of focal adhesions and actin stress fibers in response to growth factors. Cell 70:389-399.

Ridley AJ, Paterson HF, Johnston CL, Diekmann D, Hall A (1992) The small GTP-binding protein rac regulates growth factor-induced membrane ruffling. Cell 70:401-410.

Riento K, Ridley AJ (2003) Rocks: multifunctional kinases in cell behaviour. Nat Rev Mol Cell Biol 4:446-456.

Safavi-Abbasi S, Wolff JR, Missler M (2001) Rapid morphological changes in astrocytes are accompanied by redistribution but not by quantitative changes of cytoskeletal proteins. Glia 36:102-115.

Schmitz AA, Govek EE, Bottner B, Van Aelst L (2000) Rho GTPases: signaling, migration, and invasion. Exp Cell Res 261:1-12.

Singh R, Wang B, Shirvaikar A, Khan S, Kamat S, Schelling JR, Konieczkowski M, Sedor JR (1999) The IL-1 receptor and Rho directly associate to drive cell activation in inflammation. J Clin Invest 103:1561-1570.

Small JV, Rottner K, Kaverina I (1999) Functional design in the actin cytoskeleton. Curr Opin Cell Biol 11:54-60.

Smilenov LB, Mikhailov A, Pelham RJ, Marcantonio EE, Gundersen GG (1999) Focal adhesion motility revealed in stationary fibroblasts. Science 286:1172-1174

Suzuki Y, Yamamoto M, Wada H, Ito M, Nakano T, Sasaki Y, Narumiya S, Shiku H, Nishikawa M (1999) Agonist-induced regulation of myosin phosphatase activity in human platelets through activation of Rho-kinase. Blood 93:3408-3417.

Uehata M, Ishizaki T, Satoh H, Ono T, Kawahara T, Morishita T, Tamakawa H, Yamagami K, Inui J, Maekawa M, Narumiya S (1997) Calcium sensitization of smooth muscle mediated by a Rho-associated protein kinase in hypertension. Nature 389:990-994.

Verhoef PA, Estacion M, Schilling W, Dubyak GR (2003) P2X7 receptordependent blebbing and the activation of Rho-effector kinases, caspases, and IL-1 beta release. J Immunol 170:5728-5738.

Voutsinos-Porche B, Bonvento G, Tanaka K, Steiner P, Welker E, Chatton JY, Magistretti PJ, Pellerin L (2003) Glial glutamate transporters mediate a functional metabolic crosstalk between neurons and astrocytes in the mouse developing cortex. Neuron 37:275-286.

Wehrle-Haller B, Imhof B (2002) The inner lives of focal adhesions. Trends Cell Biol 12:382-389. 